\title{
THE SECOND-GENERATION $z$ (REDSHIFT) AND EARLY UNIVERSE SPECTROMETER. I. FIRST-LIGHT OBSERVATION OF A HIGHLY LENSED LOCAL-ULIRG ANALOG AT HIGH- $z$
}

\author{
Carl Ferkinhoff $^{1}$, Drew Brisbin ${ }^{1}$, Stephen Parshley ${ }^{1}$, Thomas Nikola ${ }^{1}$, Gordon J. Stacey ${ }^{1}$, Justin Schoenwald ${ }^{1}$, \\ James L. Higdon ${ }^{2}$, Sarah J. U. Higdon ${ }^{2}$, Aprajita Verma ${ }^{3}$, Dominik Riechers ${ }^{1}$, Steven Hailey-Dunsheath ${ }^{4}$, Karl \\ M. Menten ${ }^{5}$, Rolf GÜsten ${ }^{5}$, Axel Wei ${ }^{5}$, Kent Irwin ${ }^{6}$, Hsiao M. Cho $^{6}$, Michael Niemack ${ }^{7}$, Mark Halpern $^{8}$, \\ Mandana Amiri ${ }^{8}$, Matthew Hasselfield ${ }^{9}$, D. V. Wiebe ${ }^{8}$, Peter A. R. Ade $^{10}$, and Carol E. Tucker ${ }^{10}$ \\ ${ }^{1}$ Department of Astronomy, Cornell University, Ithaca, NY 14853, USA; carl.ferkinhoff@ cornell.edu \\ ${ }^{2}$ Department of Physics, Georgia Southern University, Statesboro, GA 30460, USA \\ ${ }^{3}$ Department of Physics, University of Oxford, Oxford, OX1 3RH, UK \\ ${ }^{4}$ California Institute of Technology, Pasadena, CA 91125, USA \\ ${ }^{5}$ Max-Planck-Institut für Radioastronomie, Auf dem Hügel 69, D-53121 Bonn, Germany \\ ${ }^{6}$ NIST Boulder, Boulder, CO 80305, USA \\ ${ }^{7}$ Department of Physics, Cornell University, Ithaca, NY 14853, USA \\ ${ }^{8}$ Department of Physics and Astronomy, University of British Columbia, Vancouver, BC, V6T 1Z1, Canada \\ ${ }^{9}$ Department of Astrophysical Sciences, Princeton University, Princeton, NJ 08544, USA \\ 10 Department of Physics and Astronomy, Cardiff University, Cardiff CF24 3AA, UK \\ Received 2013 August 16; accepted 2013 November 5; published 2013 December 19
}

\begin{abstract}
We recently commissioned our new spectrometer, the second-generation $z$ (Redshift) and Early Universe Spectrometer (ZEUS-2) on the Atacama Pathfinder Experiment telescope. ZEUS-2 is a submillimeter grating spectrometer optimized for detecting the faint and broad lines from distant galaxies that are redshifted into the telluric windows from 200 to $850 \mu \mathrm{m}$. It uses a focal plane array of transition-edge sensed bolometers, the first use of these arrays for astrophysical spectroscopy. ZEUS-2 promises to be an important tool for studying galaxies in the years to come because of its synergy with Atacama Large Millimeter Array and its capabilities in the short submillimeter windows that are unique in the post-Herschel era. Here, we report on our first detection of the [C II] $158 \mu \mathrm{m} \mathrm{line}$ with ZEUS-2. We detect the line at $z \sim 1.8$ from H-ATLAS J091043.1-000322 with a line flux of (6.44 \pm 0.42$)$ $\times 10^{-18} \mathrm{~W} \mathrm{~m}^{-2}$. Combined with its far-IR luminosity and a new Herschel-PACS detection of the [O I] $63 \mu \mathrm{m}$ line, we model the line emission as coming from a photo-dissociation region with far-ultraviolet radiation field, $G \sim 2 \times$ $10^{4} G_{0}$, gas density, $n \sim 1 \times 10^{3} \mathrm{~cm}^{-3}$ and size between $\sim 0.4$ and $1 \mathrm{kpc}$. On the basis of this model, we conclude that H-ATLAS J091043.1-000322 is a high-redshift analog of a local ultra-luminous IR galaxy; i.e., it is likely the site of a compact starburst caused by a major merger. Further identification of these merging systems is important for constraining galaxy formation and evolution models.
\end{abstract}

Key words: galaxies: active - galaxies: high-redshift - galaxies: individual (H-ATLAS J091043.1-000322) galaxies: starburst - submillimeter: galaxies

Online-only material: color figures

\section{INTRODUCTION}

The submillimeter view of the early universe is rapidly evolving. Improvements in instrumentation capabilities over the past $15 \mathrm{yr}$ have revealed the submillimeter band $(\sim 200-850 \mu \mathrm{m})$ as a unique probe of star formation in the early universe. These studies were launched by small to modest-sized ground-based surveys, largely of one or two colors, and moderate angular resolution $\left(\sim 10^{\prime \prime}\right)$ far exceeding the previous submillimeter surveys of cosmology experiments (e.g., Smail et al. 1997; Barger et al. 1999; Hughes et al. 1998; Weiß et al. 2009; Coppin et al. 2006). Herschel expanded these studies to include both larger scales and multiple colors. From the ground, we began highredshift spectroscopic surveys of far-infrared (IR) fine-structure lines in the 350 and $450 \mu \mathrm{m}$ telluric windows with our firstgeneration $z$ (redshift) and Early Universe Spectrometer (ZEUS1) on the Caltech Submillimeter Observatory ([C II] - HaileyDunsheath et al. 2010; Stacey et al. 2010; Brisbin et al. 2013; [O III]_Ferkinhoff et al. 2010; [N II]—Ferkinhoff et al. 2011). The Herschel-SPIRE Fourier transform spectrometer enabled similar submillimeter spectroscopy that was unobscured by telluric absorption. With the advent of the Atacama Large Millimeter Array (ALMA), studies at wavelengths between 0.43 and $3 \mathrm{~mm}$ are now making great leaps forward in terms of sensitivity and spatial resolution. However, because Herschel is no longer operating and ALMA has yet to open the $350 \mu \mathrm{m}$ (band 10) window, there are limited opportunities to detect far-IR lines from high-redshift systems at wavelengths shorter than $\sim 420 \mu \mathrm{m}$. For the [C II] $158 \mu \mathrm{m}$ line, typically the brightest FIR fine-structure line, this means ALMA can observe galaxies no closer than $z \sim 1.7$ until band 10 is deployed.

We have recently commissioned our new instrument, the second-generation $z$ (Redshift) and Early Universe Spectrometer (ZEUS-2) on the Atacama Pathfinder Experiment (APEX) in Chile. ${ }^{11}$ When fully deployed, ZEUS-2 will have wavelength coverage between 200 and $850 \mu \mathrm{m}$. For its first run, reported here, ZEUS-2 only accessed the 350 and $450 \mu \mathrm{m}$ telluric windows. With a spectral resolving power of $\sim 1000$ and background limited detectors, ZEUS-2 is optimized for detecting broad lines from galaxies (line-widths of $\sim 200-300 \mathrm{~km} \mathrm{~s}^{-1}$ ). Within these windows, ZEUS-2 can rapidly survey high-redshift galaxies, detecting far-IR lines that are spatially unresolved in a typical

\footnotetext{
11 This publication is based on data acquired with the Atacama Pathfinder Experiment (APEX). APEX is a collaboration between the

Max-Planck-Institut fur Radioastronomie, the European Southern Observatory, and the Onsala Space Observatory.
} 
ZEUS-2/APEX beam $\left(\sim 8^{\prime \prime}\right)$ yet suitable for follow-up at high spatial resolution with ALMA. In local systems ZEUS-2 can simultaneously map their emission in the ${ }^{13} \mathrm{CO} 6-5$, CO 7-6, [N II] $205 \mu \mathrm{m},\left[\mathrm{CI}_{\mathrm{I}}\right] 370 \mu \mathrm{m}$, and the [C I] $605 \mu \mathrm{m}$ lines, which provide important information about the ionized, atomic, and molecular phases of the interstellar medium (ISM). Part II of this paper (C. Ferkinhoff et al. 2013, in preparation) discusses the instrument design and performance in detail.

The star formation and ISM of high- $z$ systems can be studied with ZEUS-2 through the FIR fine-structure lines of carbon, oxygen, nitrogen, and their various ions that are redshifted into the submillimeter atmospheric windows. These lines characterize both the physical conditions of the gas and the ambient radiation fields. They are especially useful for studying dusty galaxies in the early universe where the commonly used optical emission line tracers of the ISM (e.g., [O II] 3726, 3729 and [O III] 4959, $5007 \AA$ ) undergo significant extinction, thereby limiting their use as astrophysical probes. The FIR fine-structure lines have been extensively studied in nearby extragalactic systems with the Kuiper Airborne Observatory, the Infrared Space Observatory (ISO), and recently with Herschel (e.g., Crawford et al. 1986; Stacey et al. 1991; Lord et al. 1996; Luhman et al. 1998; Malhotra et al. 2001; Negishi et al. 2001; Brauher et al. 2008; Graciá-Carpio et al. 2011). These studies were extended to high-redshift galaxies with Herschel (cf. Ivison et al. 2010; Sturm et al. 2010; Valtchanov et al. 2011; Coppin et al. 2012; A. Verma et al. 2013, in preparation) and regularly detected from the ground with ZEUS-1, as mentioned earlier, as well as with telescopes such as the IRAM $30 \mathrm{~m}$, the Plateau de Bure Interferometer (PdBI), and ALMA (e.g., Maiolino et al. 2005, 2009; Iono et al. 2006; Swinbank et al. 2012; De Breuck et al. 2011; Cox et al. 2011; DeCarli et al. 2012; Carilli et al. 2013; Wang et al. 2013). Most recently, ALMA and the PdBI have enabled the spatially resolved studies of these important cooling lines (Wagg et al. 2012; Gallerani et al. 2012).

\subsection{Studies of Far-IR [C II] and [O I] Emission}

In nearby systems, the [C II] $158 \mu \mathrm{m}$ and [O I] $63 \mu \mathrm{m}$ lines are typically the brightest of the FIR lines, with line luminosities between $\sim 0.1 \%$ and $1 \%$ of the FIR luminosity (e.g., Crawford et al. 1986; Stacey et al. 1991; Malhotra et al. 2001). These lines arise in warm and dense photo-dissociation regions (PDRs) that are formed on the surfaces of molecular clouds (Tielens \& Hollenbach 1985) by far-ultraviolet radiation (FUV; $6 \mathrm{eV}<$ $h v<13.6 \mathrm{eV}$ ) from early-type stars. The gas in PDRs is heated by electrons that are photo-ejected off small dust grains by the FUV field and cooled by the [C II] and [O I] lines. The relative strength of the lines depends on both the gas density and strength of the FUV field where the [OI] $63 \mu \mathrm{m}$ line dominates the cooling at higher densities and FUV fields. Over the typical range of PDR gas densities for extragalactic sources $(n \sim$ $10^{3}-10^{5} \mathrm{~cm}^{-3}$ ), the [C II]/FIR ratio is inversely proportional to the strength of the FUV field, so that it indicates the strength of the FUV field and source size (Stacey et al. 2010).

ISO studies found a decline in the $[\mathrm{C} \mathrm{II}] / \mathrm{FIR}$ ratio for increasingly luminous, nearby galaxies (Malhotra et al. 2001; Luhman et al. 2003). The decline is attributed to dust grains acquiring greater positive charge resulting from photo-ejection of electrons in the extreme FUV environment of the compact and intense star-forming regions that are induced by major mergers. The accumulated grain charge reduces the gas-heating efficiency of the ejected electron, limiting the collisional excitation rate of ionized carbon and tempering the rise in [C II] line emission. As a result, in clouds with densities near or higher than the critical density of the transition $\left(\sim 2 \times 10^{3} \mathrm{~cm}^{-3}\right)$ that are also exposed to high FUV fields, the excitation of the [C II] line emitting level effectively saturates, and higher gas densities and temperatures do not lead to a larger population in the excited level. The column density of $\mathrm{C}^{+}$in such environments is determined by dust extinction of the UV photons capable of ionizing carbon, so that the $\mathrm{C}^{+}$column grows only logarithmically with FUV flux. Meanwhile, the FIR emission continues to increase linearly for increasing FUV fields as a result of the dust reprocessing of the UV photons (Stacey et al. 2010). This explanation is also supported by the suppression of all of the FIR finestructures lines in the FIR luminous systems seen in the study by Graciá-Carpio et al. (2011). In some ULIRGs, however, there may be a significant active galactic nucleus (AGN) contribution to the FIR luminosity significantly reducing the $[\mathrm{C}$ II]/FIR line ratio if the AGN contribution to the FIR luminosity is not accounted for (Sargsyan et al. 2012).

The first [ $\left.\mathrm{C}_{\mathrm{II}}\right]$ survey of high- $z$, FIR luminous systems $\left(L_{\mathrm{FIR}}>10^{12.5} L_{\odot}\right)$ showed that star-formation-dominated galaxies in the early universe did not have the low [C II]/FIR ratios characteristic of the ultra-luminous systems nearby (Stacey et al. 2010). It is surprising that their ratios were similar to local moderate-luminosity systems indicating kiloparsecscale and moderate-intensity star formation-not the concentrated and merger-induced starbursts seen in local ULIRGs. The high- $z$ systems with low [C II]/FIR ratios, similar to the ratios of local ULIRGs, have an AGN that dominates their energetics (Stacey et al. 2010). In this work, we report on observations of a lensed ULIRG that we conclude is a high- $z$ analogue of local ULIRGs: a system with strong FUV fields and compact, sub-kiloparsec star formation induced by a major merger.

\subsection{This Paper: First ZEUS-2 Detection of a Spectral Line from a High-redshift Galaxy}

In this paper, we report on the first light spectra of ZEUS-2 on APEX obtained in 2012 November. We strongly $(\sim 12 \sigma)$ detect the [C $\left.{ }_{\text {II }}\right] 158 \mu \mathrm{m}$ line from H-ATLAS J091043.1-000322 in $\sim 66$ minutes of integration time. For our analysis, we combine our [C $\mathrm{CI}]$ detection with a recent Herschel detection of the [O I] $63 \mu \mathrm{m}$ line (A. Verma et al. 2013, in preparation), previous to CO detections (Lupu et al. 2012), and archival Hubble Space Telescope (HST) images. This work is the first spectroscopic use of a transition-edged sensed bolometer-array, the state-of-theart in submillimeter detector technology, and demonstrates the scientific potential of ZEUS-2.

In Section 2 of this paper, we describe the source, the ZEUS-2 observations, data reduction, and a gravitational lensing model on the basis of archival HST Images. Section 3 presents a combined analysis of the new spectroscopic measurements. The implications of this analysis are discussed in Section 4. Last, we give our concluding remarks in Section 5. Throughout this paper, we adopt the cosmological parameters of $\Omega_{\Lambda}=0.73, \Omega_{m}=0.27$ and $H_{0}=71 \mathrm{~km} \mathrm{~s}^{-1} \mathrm{Mpc}^{-1}$ (Spergel et al. 2003). We define the far-IR luminosities to be from 42.5 to $122.5 \mu \mathrm{m}$ (covering the IRAS 60 and $100 \mu \mathrm{m}$ bands) following the prescription of Helou et al. (1988). Some authors extend the FIR luminosity to include wavelengths up to $500 \mu \mathrm{m}$; these values are typically $\sim 1.5$ times larger than the luminosities we report here. The total IR luminosity, $L_{\mathrm{IR}}$, is the integrated luminosity between 8 and $1000 \mu \mathrm{m}$ and is $\sim 2$ times larger than the FIR luminosity as we define earlier. 
Table 1

H-ATLAS J091043.1-000322 Source Parameters

\begin{tabular}{|c|c|c|c|}
\hline Parameter & Unit & Value & Source \\
\hline R.A. & hh:mm:ss.s & $9: 10: 43.1$ & Lupu et al. (2012) \\
\hline Decl. & dd:mm:ss & $-00: 03: 22$ & Lupu et al. (2012) \\
\hline$Z$ & $\mu \cdot 10^{-18} \mathrm{~W} \mathrm{~m}^{-2}$ & 1.786 & Negrello et al. (2010) \\
\hline$\mu$ & $\mu \cdot 10^{-18} \mathrm{~W} \mathrm{~m}^{-2}$ & $18 \pm 11$ & This work \\
\hline$L_{\mathrm{FIR}}$ & $\mu \cdot 10^{13} L_{\odot}$ & $3.88 \pm 0.47$ & This work \\
\hline$M_{\mathrm{H} 2}$ & $\mu \cdot 10^{11} M_{\odot}$ & 2.0 & Lupu et al. (2012) \\
\hline$\mu \cdot \Omega_{d}$ & $\operatorname{arcsec}^{2}$ & 0.43 & Lupu et al. (2012) \\
\hline$F([\mathrm{C}$ II $] 158)$ & $\mu \cdot 10^{-18} \mathrm{~W} \mathrm{~m}^{-2}$ & $6.44 \pm 0.42$ & This work \\
\hline$F\left(\left[\mathrm{O}_{\mathrm{I}}\right] 63\right)$ & $\mu \cdot 10^{-18} \mathrm{~W} \mathrm{~m}^{-2}$ & $7.5 \pm 2.3$ & A. Verma et al. (in preparation) \\
\hline $\mathrm{CO}(2-1)$ & $\mu \cdot 10^{-18} \mathrm{~W} \mathrm{~m}^{-2}$ & $0.0234 \pm 0.00257$ & D. A. Riechers et al. (in preparation) \\
\hline $\mathrm{CO}(5-4)$ & $\mu \cdot 10^{-18} \mathrm{~W} \mathrm{~m}^{-2}$ & $0.159 \pm 0.053$ & Lupu et al. (2012) \\
\hline $\mathrm{CO}(6-4)$ & $\mu \cdot 10^{-18} \mathrm{~W} \mathrm{~m}^{-2}$ & $0.241 \pm 0.083$ & Lupu et al. (2012) \\
\hline $\mathrm{CO}(7-6)$ & $\mu \cdot 10^{-18} \mathrm{~W} \mathrm{~m}^{-2}$ & $0.174 \pm 0.136$ & Lupu et al. (2012) \\
\hline$\left[\mathrm{C}_{\mathrm{I}}\right] 370$ & $\mu \cdot 10^{-18} \mathrm{~W} \mathrm{~m}^{-2}$ & $0.301 \pm 0.136$ & Lupu et al. (2012) \\
\hline
\end{tabular}

\section{THE SOURCE, OBSERVATIONS, AND LENSING MODEL}

\subsection{H-ATLAS J091043.1-000322}

First reported by Negrello et al. (2010), H-ATLAS J091043.1-000322 (hereafter SDP11) was identified in the Herschel guaranteed time program Herschel Astrophysical Terahertz Large Area Survey (H-ATLAS; Eales et al. 2010). Subsequent spectroscopic follow-up with $Z$-Spec on the CSO identified several mid- $J$ CO transitions at $z=1.786$ (Lupu et al. 2012). On the basis of its exceptionally bright line fluxes, its very large apparent FIR luminosity ${ }^{12}\left(L_{\mathrm{FIR}}=3.88 \times 10^{13} L_{\odot}\right)$ and the identification of a foreground lensing galaxy at $z=$ 0.793 , Negrello et al. conclude that SDP11 is a highly gravitationally lensed system, with more moderate intrinsic luminosity. Negrello et al. do not, however, constrain the magnification. Using archival $H S T$ observations and a simple lensing model, we confirm that SDP11 is a lensed galaxy with magnification, $\mu$, between 7 and 29 (see Section 2.3), so that the intrinsic molecular-gas mass and far-IR luminosity are $\sim(0.8-3.4) \times$ $10^{10} M_{\odot}$ and $\sim(1.3-5.4) \times 10^{12} L_{\odot}$, respectively. Table 1 lists the observed source properties from this work and the literature.

\subsection{Observations}

Using ZEUS-2 on APEX, we observed SDP11 on 2012 November 17 under very good observing conditions $(0.48 \mathrm{~mm}$ of precipitable water vapor). Spectra were obtained in standard chopping and beam switching mode with a chopper throw of $60^{\prime \prime}$. We obtained five 13.2 minute integrations for a total integration time of 66 minutes. The ZEUS-2 velocity resolution at the observed wavelength $(439.6 \mu \mathrm{m})$ is $\sim 340 \mathrm{~km} \mathrm{~s}^{-1}$. The ZEUS-2/APEX beam is $8^{\prime \prime}$ ( $\sim 68 \mathrm{kpc}$ at $z=1.78$ ), as measured on Uranus, which fully contains SDP11. Uranus also served as our flux calibrator using a brightness temperature of $68 \mathrm{~K}$ at $450 \mu \mathrm{m}$, as reported by Hildebrand et al. (1985). Figure 1 shows the detected [C II] $158 \mu \mathrm{m}$ line with a flux of $(6.44 \pm 0.42) \times$ $10^{-18} \mathrm{~W} \mathrm{~m}^{-2}$.

\footnotetext{
12 Negrello et al. (2010) and Lupu et al. (2012) report different values for the FIR luminosities of SDP11 likely due to different definitions of the FIR luminosities. To ensure what we are consistent with our ZEUS-1 sample in Brisbin et al (2013) we have taken the photometry reported in Negrello et al. and performed our own fit of the spectral energy distribution using the spectral energy distribution templates of Siebenmorgen \& Krügel (2007) and integrating the spectral energy distribution according to our definition of the FIR luminosity in Section 1.2.
}

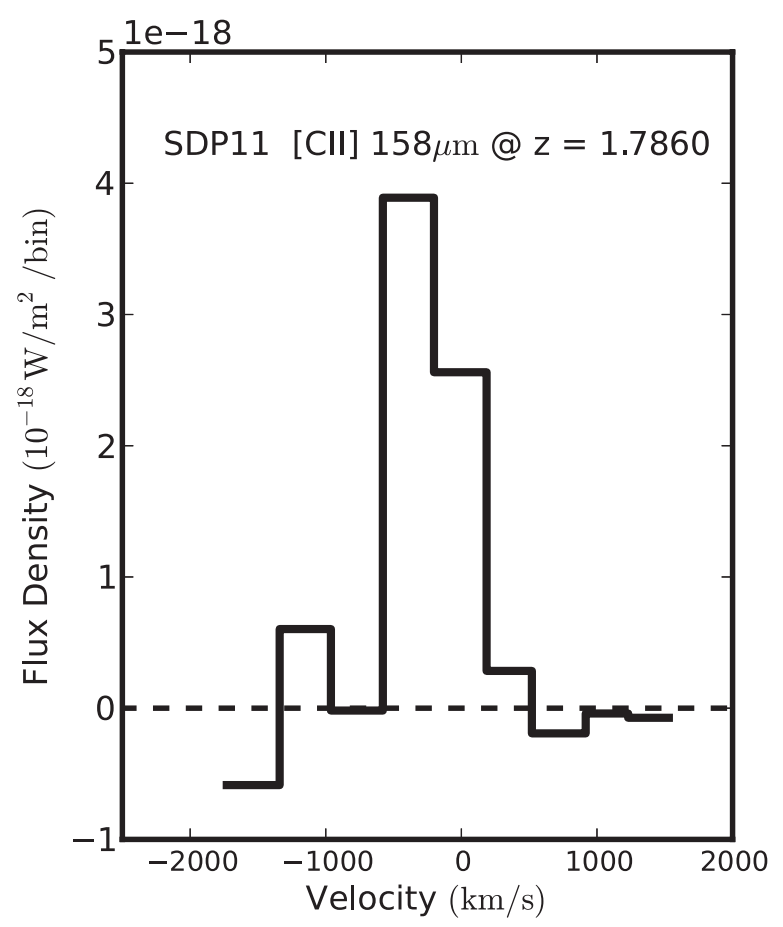

Figure 1. ZEUS-2/APEX detection of the [C II] $158 \mu \mathrm{m}$ line from H-ATLAS J091043.1-000322 plotted vs. the source rest-frame velocity. Spectral bins are $\sim 1$ resolution element and equal to $\sim 350 \mathrm{~km} \mathrm{~s}^{-1}$. The continuum emission has been removed.

\subsection{Lensing Model}

Figure 2 shows a Hubble Wide-Field Camera 3 (WFC3) near-IR image (F110W) of SDP11, centered on the lensing galaxy SDSS J091043-000323 (hereafter SDSSJ0910) at $z=0.793$ (central elliptical at position $\left.0^{\prime \prime}, 0^{\prime \prime}\right)$. The contours show the $H S T$ /WFC3 F160W image divided by the F110W image, i.e., the $1.5 \mu \mathrm{m} / 1.1 \mu \mathrm{m}$ image. ${ }^{13}$ On the basis of the spectral energy distribution modeling of Negrello et al. (2010), who separately fit a spectral energy distribution to SDP11 and the lensing galaxy SDSSJ0910, we expect both SDSSJ0910 and SDP11 to be brighter in the $1.5 \mu \mathrm{m}$ band. However, the $1.5 / 1.1 \mu \mathrm{m}$ ratio for SDP11 is $\sim 4$ times larger than that of SDSSJ0910, and the

\footnotetext{
13 These images were obtained from the Hubble Legacy Archive and where part of $H S T$ cycle 18 proposal 12,194 and are described in detail in Negrello et al. (2013) and Dye et al. (2013).
} 


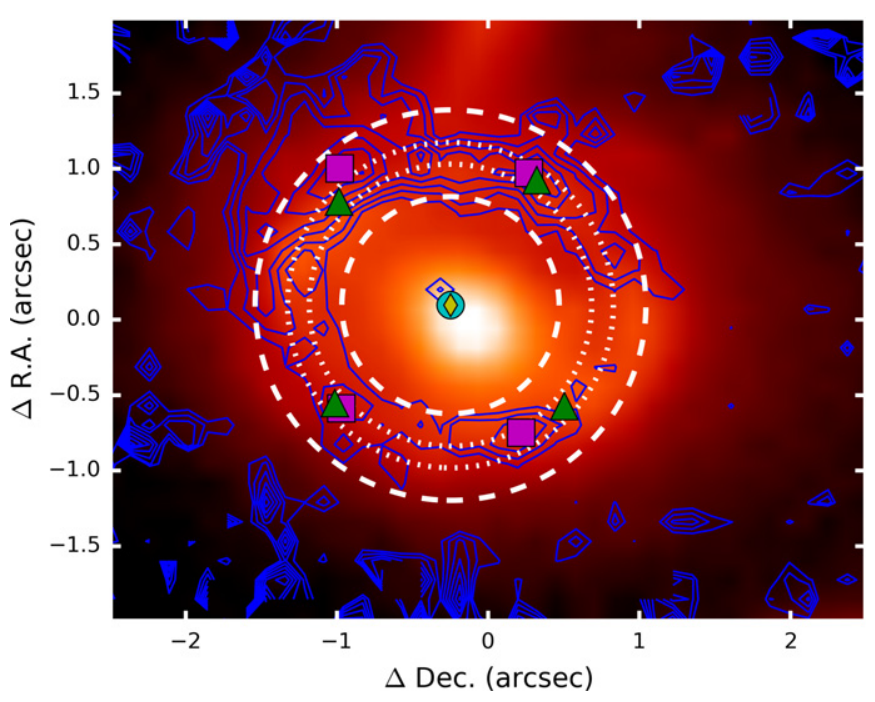

Figure 2. HST/WFC3 F110W image with F160W/F110W divided image overlaid in blue contours. The squares denote the positions of the peaks in the divided image used for the gravitational lens model described in Section 2.3. The triangles show the best-fit positions of the emission peaks, and the diamond locates the best-fit lens and source positions. The white contours are the $70 \%$ of the peak-flux limits for Einstein rings produced by our best-fit lens model and an extended source with Gaussian light profile and half-light diameters of $0.7 \mathrm{kpc}$ (dotted) and $3.4 \mathrm{kpc}$ (dashed).

(A color version of this figure is available in the online journal.)

divided image should show a lensed imaged of SDP11. The divided image (i.e., the contours in Figure 2) clearly shows an Einstein ring centered on SDSSJ0910. To create a lens model, we take the positions of the ring's peak values relative to the centroid of SDSSJ0910 to correspond to lensed images of SDP11 assuming it is a point source. We input the position and values of the peaks in the divided image into LENSMODEL (Keeton 2001) to model the gravitational lens system and reproduce the peaks of the Einstein ring. The squares indicate the peak positions, and the triangles indicate the predicted image locations on the basis of our model solution. While we obtain an excellent model fit to the image positions and fluxes $\left(\tilde{\chi}^{2} \sim 1\right)$, to determine the magnification and reproduce the observed Einstein ring, we use the point-source-derived model of the lens but replace the point source with an extended source. Replacing with an extended source adds uncertainty to the magnification factor because we do not know how well the width of the ring in the divided image represents the true source size. With that caveat in mind, a source with a Gaussian light profile and half-light radius of $2.1 \pm 1.3 \mathrm{kpc}$ is able to reproduce the observed width of the Einstein ring in Figure 2 and results in a flux magnification, $\mu$, of $18 \pm 11$. This is the magnification of the NIR emission in SDP11. The magnification of SDP11's submillimeter emission could be different if it is distributed differently relative to the position of the lens. Given the lack of high-resolution submillimeter images, we adopt the NIR magnification above throughout this paper with the caveat that the submillimeter magnification may be different.

\section{ANALYSIS}

\subsection{The [C II] $158 \mu \mathrm{m}$ to FIR Luminosity Ratio: Intense FUV Fields}

SDP11 has an apparent FIR luminosity of $3.88 \times 10^{13} L_{\odot}$, placing it firmly in the hyper-luminous IR galaxy class (HyLIRG, $L_{\mathrm{FIR}} \geqslant 10^{3} L_{\odot}$ ). Figure 3 shows a plot of the $L_{[\mathrm{CII}]} /$

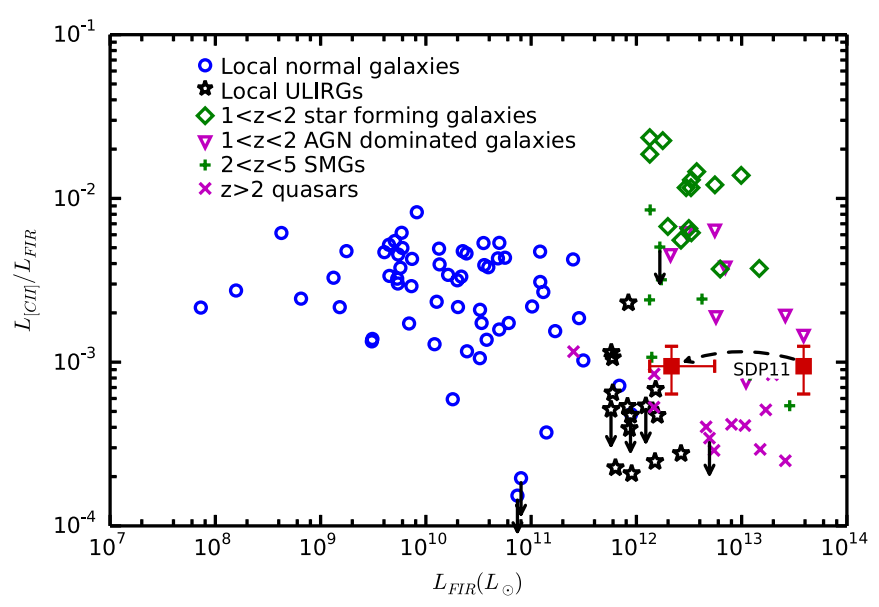

Figure 3. $L_{[\mathrm{CII}]} / L_{\mathrm{FIR}}$ ratio as a function of the $L_{\mathrm{FIR}}$ for local and high-redshift galaxies. The ratio from SDP11 (square) is plotted versus both its apparent and intrinsic FIR luminosity. The effect of delensing SDP11is noted with an arrow, and the horizontal error bars indicate the effects of the allowed magnification factors. For comparison, we include ratios for local normal star-forming galaxies (Malhotra et al. 2001), local ULIRGs (Luhman et al. 2003), the ZEUS-1 star forming and AGN samples (Stacey et al. 2010; Brisbin et al. 2013; S. HaileyDunsheath et al. 2013, in preparation), $z>2$ submillimeter bright galaxies (Maiolino et al. 2009; Ivison et al. 2010; De Breuck et al. 2011; Swinbank et al. 2012; Wagg et al. 2012; Valtchanov et al. 2011; Riechers et al. 2013), and $z>$ 2 quasars (Pety et al. 2004; Maiolino et al. 2005, 2009; Gallerani et al. 2012; Wagg et al. 2012; Carilli et al. 2013; Maiolino et al. 2005; Leipski et al. 2013; Willott et al. 2013; Venemans et al. 2012).

(A color version of this figure is available in the online journal.)

$L_{\text {FIR }}$ ratio, $R$, for SDP11 versus its apparent FIR luminosity along with samples of local galaxies-normal star-forming galaxies and local ULIRGs - and high- $z$ galaxies including the sources from Stacey et al. (2010) and Hailey-Dunsheath et al. (2010). The ratio $R \sim(1.0 \pm 0.3) \times 10^{-3}$ for SDP11 falls between the average line ratios observed for star-formation-dominated systems $\left(L_{[\mathrm{CII}]} / L_{\mathrm{FIR}} \sim 3 \times 10^{-3}\right)$ and AGN-dominated systems $\left(L_{[\mathrm{CII}]} / L_{\mathrm{FIR}} \sim 4 \times 10^{-4}\right)$ at high- $z$. Stacey et al. (2010) and Hailey-Dunsheath et al. (2010) show that stronger UV fields may lower the value of $R$ and for a given value of $R$, i.e., for a given FUV field, the FIR luminosity indicates the spatial scale of the emission. The ratio of SDP11, when compared with the Stacey et al. sample of sources with similar ratios, suggests a FUV field, $G$, of $\sim 10,000 G_{0}$, where $G_{0}$ is the Habing field-the strength of the local interstellar radiation field corresponding to a flux of $1.6 \times 10^{-3} \mathrm{erg} \mathrm{cm}^{-2} \mathrm{~s}^{-1}$. Given the value of $R$ from SDP11, its FIR luminosity suggests a source size greater than a kilo-parsec. However, once we account for the magnification of SDP11, its intrinsic FIR luminosity becomes similar to local ULIRGS. Magnification does not affect the estimate of the FUV field $\left(G \sim 10,000 G_{0}\right)$, which is more intense than local normal galaxies or high- $z$ star-formation-dominated systems, but the lower FIR luminosity now suggests sub-kiloparsec size emission similar to that found in nearby ULIRGs.

\subsection{Modeling the Line and Continuum Emission}

Comparisons between our [C II] line, the [O I] line, and the FIR continuum will provide tighter constraints on the ambient FUV fields and gas density in SDP11 as well as the nature of the source. Using the Herschel-PACS spectrometer on the Herschel Space Telescope, A. Verma et al. (2013, in preparation) report a [O I] $63 \mu \mathrm{m}$ line flux of $(7.5 \pm 2.3) \times 10^{-18} \mathrm{~W} \mathrm{~m}^{-2}$. Typically, the [C II] and [OI] lines are produced in PDRs associated with star-forming regions. However, it is possible 


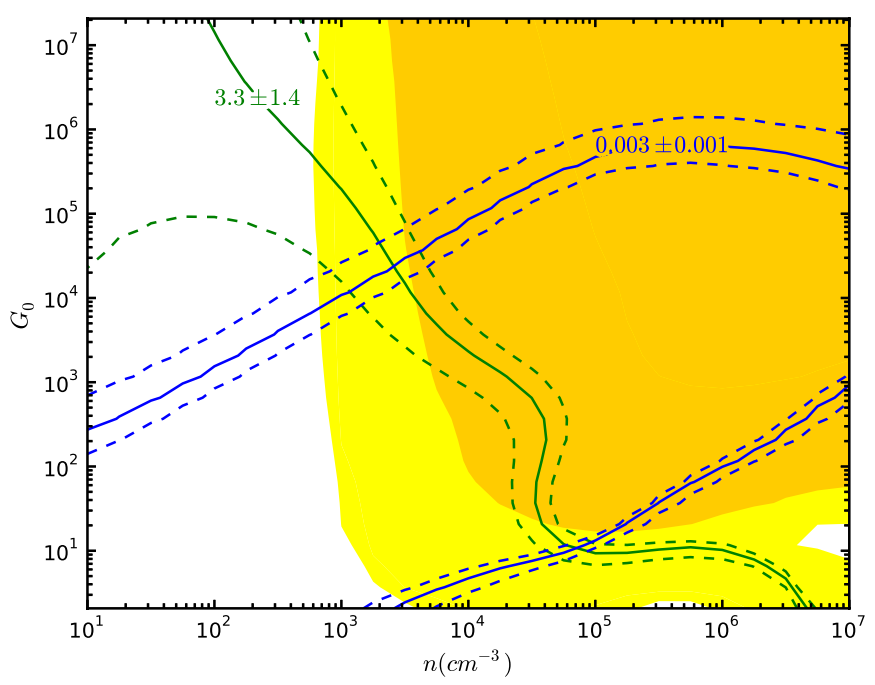

Figure 4. PDR modeling using the online PDR Toolkit (Pound \& Wolfire 2008; Kaufman et al. 2006) of the corrected [O I $] /[\mathrm{C} \mathrm{II}]$ ratio (green), ([O I $]+[\mathrm{C} \mathrm{II}]) /$ FIR ratio (blue) from SDP11. The shaded regions show the allowed $G_{0}$ and $n$ phase space of the mid- $J$ CO observations of Lupu et al. 2012 where yellow is the ratio of $\left[\mathrm{C}_{\mathrm{I}}\right] 370 \mu \mathrm{m}+\mathrm{CO}(7-6)$ to $\mathrm{CO}(5-4)$ and orange is the $\mathrm{CO}(6-5) /$ $\mathrm{CO}(5-4)$ ratio. The dash lines indicate $\pm 1 \sigma$ error bounds of the ratios listed earlier. For all lines, we have corrected for ionized gas and optical depth effects as described in the text.

(A color version of this figure is available in the online journal.)

that both of these lines arise within the X-ray-dominated regions produced in molecular clouds enveloping an AGN. Meijerink et al. (2006) produce grids of observed flux for various FIR fine-structure lines, including [C $\mathrm{II}]$ and $\left[\mathrm{O}_{\mathrm{I}}\right]$, across typical densities and X-ray fluxes of X-ray-dominated regions. The observed $[\mathrm{OI}] /[\mathrm{C} \mathrm{II}]$ ratio is $1.2 \pm 0.5$. This is consistent only with the lowest X-ray fluxes and densities $\left(F_{X} \sim 0.1-0.3 \mathrm{erg}\right.$ $\mathrm{cm}^{-2} \mathrm{~s}^{-1}$ and $\left.n \sim 100-1000\right)$, so we expect that the line emission from SDP11 is likely not dominated by an X-raydominated region associated with an AGN. Furthermore, since the currently available photometry of SDP11 can be fit solely by a star-formation-dominated spectral energy distribution (see Lupu et al. 2012), we proceed with an analysis of the observed $[\mathrm{C} I \mathrm{I}]$ and $[\mathrm{OI}]$ lines and far-IR continuum emission within a star-formation-dominated PDR paradigm.

Kaufman et al. (2006) produce model grids of PDR regions over a range of densities and FUV fields, which we use for our modeling. In performing the PDR modeling, we assumed $\sim 70 \%$ of the observed [C II] flux arise in the PDR, with most of the remaining $30 \%$ arising from the warm ionized medium (Oberst et al. 2006). The models of Kaufman et al. assume a single face-on cloud. In reality, the PDRs in SDP11 are more complex than used in Kaufman et al., and if we assume that they are spherical, then we will detect [C II] and FIR emission from both the front and the back of the cloud, while we only see [O I] from the front because it is optically thick. To make use of the Kaufman et al. models, we must then multiply our observed [O I] line flux by two to account for the emission that can be selfabsorbed along the line of sight by intervening atomic oxygen. Both of these corrections are appropriate for analysis within a star-formation paradigm (Kaufman et al. 1999). In Figure 4, we plot the corrected $[\mathrm{C} \mathrm{II}] / \mathrm{FIR}$ and $([\mathrm{C} \mathrm{II}]+[\mathrm{OI}]) / F I R$ flux ratios for SDP11. This gives a PDR model solution of $G=$ $20,000 G_{0}$ and a gas density $n=2500 \mathrm{~cm}^{-3}$. In principle, CO observations are also useful in constraining the PDR model. The observed CO lines are in agreement with our [C II $],\left[\mathrm{O}_{\mathrm{I}}\right]$, and
FIR-constrained model; however, because of the low signal-tonoise detection of these lines from SDP11, their inclusion does not help to constrain it further, as is evident by Figure 4.

Our derived FUV field and gas density are consistent with values observed in both local ULIRGs (e.g., Luhman et al. 2003) and high- $z$ sources dominated by AGN (e.g., Stacey et al. 2010), agreeing with our conclusions above that were drawn from the [C II] to continuum ratio alone. While the observations of the [C II], [OI], and FIR alone are not enough to unambiguously identify the nature of the source, describing their luminosities entirely within a star-formation paradigm suggests SDP11 most likely features a compact starburst similar to those in local ULIRGs. While this does not exclude the presence of an AGN, if one is present in SDP11 it likely does not contribute significantly to our observations or conclusions.

Having constrained the FUV field within SDP11, we estimate the size of the emission region, i.e., the size of the starburst, following the method from Stacey et al. (2010). This will provide additional clues to the nature of the source. Since the FIR emission arises from processing of the FUV radiation by dust, the ratio of the FIR luminosity to the FUV radiation field determines the size of the emitting regions. From Wolfire et al. (1990), the source diameter, $D$, is proportional to $\left(\lambda L_{F I R} / G\right)^{1 / 3}$ if the mean-free path $(\lambda)$ of a FUV photon is small. If instead the mean-free path of a FUV photon is large, then $D \propto\left(L_{F I R} / G\right)^{1 / 2}$. The constant of proportionality of these relations is determined by assuming that the mean-free path in SDP11 is the same for M82, which has $D \sim 300$ pc (Joy et al. 1987), $G \sim 1000 G_{0}$ (Lord et al. 1996), and $L_{\mathrm{FIR}} \sim 2.8 \times 10^{10} L_{\odot}$ (the average of the values reported in Colbert et al. 1999, and Rice et al. 1988). The source diameter is then between 0.4 and $0.9 \mathrm{kpc}$ if we assume that SDP11 is lensed a factor between 7 and 29, and we account for the extremes in mean-free path as described earlier. This agrees with the size of the dust emission determined in Lupu et al. (2012) who estimate the solid angle, $\mu \Omega_{d}$, of the dust emission region in SDP11 to be $0.43 \operatorname{arcsec}^{2}$. Accounting for the magnification by gravitational lensing, this corresponds to a circular area with diameter between 0.6 and $1.36 \mathrm{kpc}$, albeit with significant caveats as described in Lupu et al. At the same time, our PDR region size is significantly smaller than the intrinsic source size obtained from our lensing model. This disagreement is not a big concern, however, and may even be expected. Because the lensing model is based on a $1.5 \mu \mathrm{m}$ image, i.e., $\sim 397 \mathrm{~nm}$ rest frame, it is sensitive to the total stellar component in SDP11. Our PDR analysis is sensitive only to the massive and young $\mathrm{O}$ and $\mathrm{B}$ stars necessary for the ionization and excitation of [C II]. As such, we expect the PDR-derived size to be smaller than our lensing model. This also implies that we cannot use our PDR source size to better constrain the results of our lensing model.

\section{DISCUSSION}

Our analysis suggests that SDP11 is a highly lensed analogue of a local ULIRG system, containing a compact star-formation region and concomitant high FUV fields, albeit in the early universe. Unfortunately, the true intrinsic source luminosity depends on our adopted magnification factor, which is not well constrained. A useful diagnostic plot that can help confirm our conclusion on the nature of SDP11 is the plot of $L_{[\mathrm{CII}]} /$ $L_{\mathrm{FIR}}$ versus $L_{\mathrm{CO}(1-0)} / L_{\mathrm{FIR}}$ as described in Stacey et al. (2010) and shown in Figure 5. Because in the star-forming paradigm these lines and the FIR continuum arise in the same regions, these ratios should be insensitive to variations in magnification 


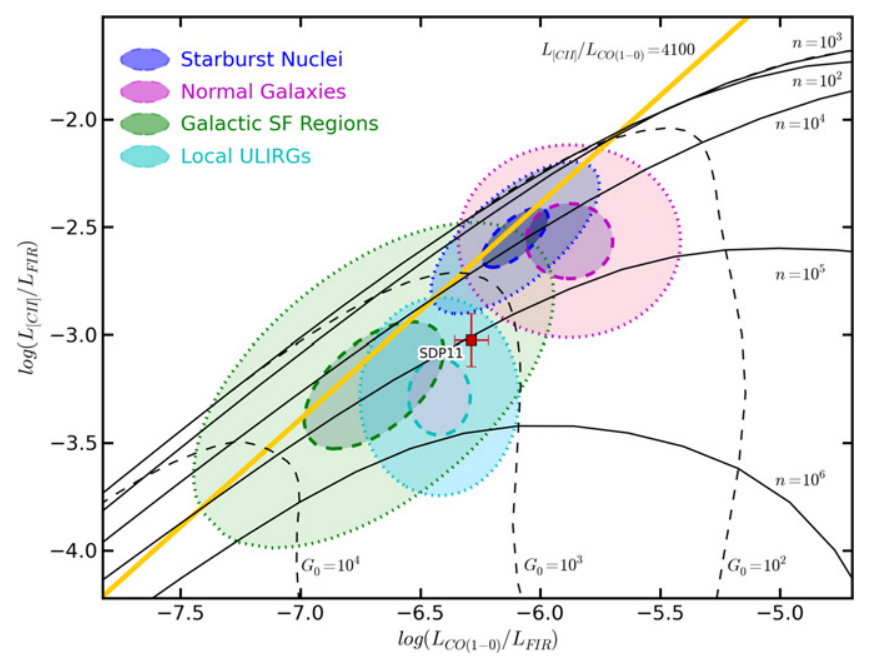

Figure 5. The $\log \left(L_{[\mathrm{CII}]} / L_{\mathrm{FIR}}\right)$ vs. $\log \left(L_{\mathrm{CO}(1-0)} / L_{\mathrm{FIR}}\right)$ for SDP11 adapted from Stacey et al. (2010). Overlaid are the FUV radiation field and gas density contours from Kaufman et al. (2006). The thick line shows the typical $L_{[\mathrm{CII}]} /$ $L_{\mathrm{CO}(1-0)}$ ratio for star-forming galaxies $(\sim 4100)$. Regions occupied by galactic star-forming regions, starburst galaxies, normal galaxies, and local ULIRGs are illustrated with $90 \%$ (dashed) and 50\% (dotted) maximum contours of twodimensional Gaussians fit to the various source samples from literature (e.g. Stacey et al. 1991; Malhotra et al. 2001; Luhman et al. 2003).

(A color version of this figure is available in the online journal.)

caused by slight difference in location of the source relative to the critical curve of the lens. In Figure 5, we identify typical regions occupied by nearby sources as well as FUV radiation and density contours from the PDR models of Kaufman et al. (2006). If a source falls in the lower right section of the plot with $[\mathrm{C} \mathrm{II}] / \mathrm{CO} \leqslant 4100$, then the observed flux to continuum ratios can be explained fully in a star-forming paradigm. Sources that fall in the upper left half of the plot may have significant [C II] emission from non-PDR sources such as X-ray-dominated regions within AGN (Stacey et al. 2010), low-density ionized gas, or low-metallicity molecular clouds (Stacey et al. 1991).

The lowest CO rotational transition detected from SDP11 is the CO 2-1 line from D. A. Riechers et al. (2013, in preparation). On the basis of the PDR model solution from Section 6, we can estimate the ratio of $\mathrm{CO}(2-1)$ to $\mathrm{CO}(1-0)$ and hence the strength of the $\mathrm{CO}(1-0)$ line. For our model-derived FUV field strength and gas density, the expected $\mathrm{CO}(2-1) / \mathrm{CO}(1-0)$ line-integrated flux ratio is 7:1, agreeing with observations of both local and high- $z$ systems (Downes \& Solomon 1998; Bradford et al. 2003; Weiß et al. 2005a, 2005b; Ward et al. 2003; Israel 2009; Aravena et al. 2008). Using this estimate along with the observed [C II] and FIR luminosity, we place SDP11 on the plot of $L_{[\mathrm{CII}]} / L_{\mathrm{FIR}}$ versus $L_{\mathrm{CO}(1-0)} / L_{\mathrm{FIR}}$. The location of SDP11 clearly places it in the ULIRG region of the plot. More generally, because it falls in the allowed region of the plot, we have further support that our analysis in the PDR paradigm and PDR derived source size are correct. As such, SDP 11 does appear analogous to local ULIRGs in terms of luminosity, FUV field strength, and source size, and it may contains a starburst produced through the interaction between two Milky-Way-sized galaxies.

To further confirm the nature of SDP11, we can ask where it falls in the gas mass-star formation relation. Genzel et al. (2010) study the relations between the star-formation rate (SFR) and the molecular gas in galaxies from $z \sim 0$ to $\sim 3$. They find that quiescent star-forming galaxies at all epochs follow a similar relation between their FIR luminosity and their CO luminosity, tracers of star formation, and molecular gas, respectively. However, merging systems - both local ULIRGs and high- $z$ mergers-lie above the sequence of quiescently star-forming galaxies, suggesting a higher star-formation efficiency in merging systems. Where is SDP11? The FIR luminosity and $\mathrm{CO}(2-1)$ luminosity scaled to the expected $\mathrm{CO}(1-0)$ luminosity as shown earlier, places SDP11 among the merging systems suggesting a mergerinduced star-formation efficiency for SDP11 higher than "normal" galaxies. Over the range of allowed magnification factors, SDP11 falls among the samples of local ULIRGS $(\mu=29)$ or high- $z$ mergers $(\mu=7)$ in the $L_{\mathrm{FIR}}-L_{\mathrm{CO}}$ plane of Genzel et al. (2010). Both of these samples lie on a $L_{\mathrm{FIR}}-L_{\mathrm{CO}}$ relation that is $\sim 4$ times that of normal galaxies.

Studies over the past decade have shown ULIRGs in the early universe to be a very diverse population. Evidence for different modes powering high- $z$ ULIRGs, including AGN, mergers, and the accretion of gas from the intergalactic medium, have all been observed. The discovery of the latter mode, however, was very surprising (cf. Biggs \& Ivison 2008; Tacconi et al. 2010; Iono et al. 2009, Hailey-Dunsheath et al. 2010; Stacey et al. 2010). In local ULIRGs, star formation is triggered by mergers of massive galaxies (Sanders \& Mirabel 1996), and it was long thought that only an AGN or similar major-merger event could produce of the extreme luminosities in high- $z$ systems. We now know the star-formation process in some $z \sim 1-3$ systems can also be stimulated through the accretion of cold gas (Tacconi et al. 2013; Genzel et al. 2010; Daddi et al. 2007). The large scale of these starbursts are best understood as Schmidt-Kennicutt law star formation, with star-forming efficiencies similar to local "normal" galaxies, but with starburst-like SFRs arising from the large molecular-gas reservoirs accreted from the cosmic Web. Studies revealing the accretion mode in high- $z$ galaxies have created a paradigm shift in our thinking about galaxy evolution and formation in the early universe. Recent models have even demonstrated that cold accretion and typical Kennicutt-type star-formation efficiencies can fully account for observed black hole growth and stellar mass assembly in the early universe (Di Matteo et al. 2012; Keres et al. 2005; Genel et al. 2012; Agertz et al. 2011; Kitzbichler \& White 2007).

This is not so say that the mergers are uncommon or unimportant in the early universe. For one, observational and theoretical evidence suggests a higher merger rate in the past (Lotz et al. 2011; Hopkins et al. 2010; Bertone \& Conselice 2009). Furthermore, the relative importance and prevalence of merger-driven star formation versus accretion-driven star formation in the early universe is still highly contested because of observational challenges in identifying merger events at high$z$ as well as measuring the full extent and size of gas disks. With the advent of high spatial-resolution submillimeter/millimeter observatories (e.g., PdBI, ALMA, and the Submillimeter Array), we are now beginning to adequately address this question.

Recently, Bothwell et al. (2013) examined the CO and FIR emission of 40 luminous $\left(L_{\mathrm{FIR}} \gtrsim 10^{12} L_{\odot}\right)$ galaxies at $z \sim$ 1.2-4.1 using the PdBI and selected for in the submillimeter (i.e., submillimeter galaxies, SMG). The authors conclude that $20 \%-28 \%$ of their sources show signs of an ongoing merger. Yet, within the observational constraints, there is no evidence suggesting increased star-formation efficiencies as one might expect for a merger and as seen in other works (e.g., Genzel et al. 2010). Another indicator of the star-formation efficiency in a galaxy is where it falls in the SFR and stellar mass relation $\left(\mathrm{SFR}-M_{*}\right)$, which has shown a tight relation between the SFR and stellar mass in normal galaxies (i.e., accretion mode), with them falling along a "main sequence" (Brinchmann et al. 2004; 
Noeske et al. 2007; Elbaz et al. 2007). Merging systems lie above the main sequence (Elbaz et al. 2011). Hung et al. (2013) examine the SFR $-M_{*}$ relation and morphology of the $0.2<z<$ $1.5 \mathrm{Herschel}$-selected galaxies. On the basis of morphological classification Hung et al. find that the fraction classified as "irregular" (indicating a merger) increases with IR luminosity at all values of $M_{*}$. Furthermore, they find that the fraction of interacting or merging systems increases with $L_{\mathrm{IR}}$ as well as distance above the main sequence, with nearly $50 \%$ of galaxies showing evidence of a merger at $L_{\mathrm{IR}}>10^{11.5} L_{\odot}$. Perhaps most interesting, however, is Hung et al.'s finding that $\gtrsim 18 \%$ of IR-luminous galaxies on the main sequence show evidence of interactions and mergers, suggesting that evolution of galaxies on the main sequence may not be explained by gas accretion and exhaustion alone.

It is clear that there is much work yet to be done in understanding the various modes of star formation and their impact in the early universe. For example, one might ask, what is the product of a major merger at early times? One possibility is that mergers, while not responsible for the bulk of star formation in the universe, are responsible for the formation of modern-day giant ellipticals. Fu et al. (2013) report on the discovery of a system, HXMM01, featuring two submillimeter galaxies in the early stages of a merger. On the basis of the gas-depletion time scales and SFRs, these authors conclude that HXMM01 may be the progenitor of a modern day elliptical. The merging galaxies in HXMM01 feature star-forming regions of $\sim 1.4 \mathrm{kpc}$ in diameter, similar to the size of the star-forming regions we estimate for SDP11. Perhaps then SDP11 represents a later stage in the merger process than HXMM01, in which the galaxies have already coalesced.

To test the various models of galaxy formation and constrain galaxy-evolution scenarios, we need to look for signs of different modes of star formation, i.e., accretion- versus merger-driven. Yet, identifying merging systems has proven to be a challenge at high- $z$. Morphological determinations such as those in Hung et al. (2013) require both high sensitivity and high spatial resolutions. Until recently, this was easiest to do at visible wavelengths, which is compromised by the large dust content of many IR luminous systems at high- $z$. Now, however, ALMA allows us to probe the morphologies of early galaxies in their rest-frame IR emission, such as the work in Fu et al. (2013) mentioned earlier, reducing the challenges of morphological classification. Using ZEUS-2, we can perform a census of the high- $z$ analogs to local ULIRGs by observing their fine-structure line emission. By combining ZEUS-2 and ALMA observations, we can morphologically calibrate various FIR fine-structure line ratios in high- $z$ systems to either merger- or accretion-driven modes of star formation instead of relying on extrapolations from local systems. This will allow us to classify the mode of star formation in systems that are spatially unresolved by ALMA. Last, in addition to assessing their total numbers, we can hope to identify the numbers of high- $z$ ULIRG analogues in their various stages of merger to provide detailed constraints on the peak of the major merger rate in the universe and the effects of mergers on galaxy properties.

\section{SUMMARY}

We have built a new submillimeter spectrometer, ZEUS-2, and recently commissioned it on the APEX telescope detecting the $[\mathrm{C}$ II $] 158 \mu \mathrm{m}$ line from SDP11 at $z \sim 1.8$. Combining our ZEUS-2 observations with a new Herschel detection of the [O I ]
$63 \mu \mathrm{m}$ line, archival HST images, and data from the literature we determine the following.

1. SDP11 is strongly gravitationally lensed with magnification factor $\mu \sim 7-29$.

2. An analysis of the [C II], [O I], and FIR emission within a PDR paradigm yields FUV fields of $G \sim 20,000 G_{0}$, gas densities of $\sim 2300 \mathrm{~cm}^{-3}$, and a source size between 0.4 and $0.9 \mathrm{kpc}$ in diameter for SDP11, all of which are similar to values seen in local ULIRGs.

3. We conclude that SDP11 is likely a high- $z$ analog of local ULIRGs featuring a compact and intense starburst confined to sub-kiloparsec scales and induced by a merger of two or more galaxies.

This work demonstrates the science that ZEUS-2 is capable of providing. Because of its synergy with ALMA and its being the only spectrometer capable of observing between 200 and $300 \mu \mathrm{ms}$ in the post-Herschel era, we expect ZEUS-2 will be an important tool for studying galaxies in the years to come.

Part of this work is based on observations made with the NASA/ESA Hubble Space Telescope, and obtained from the Hubble Legacy Archive, which is collaboration between the Space Telescope Science Institute (STScI/NASA), the Space Telescope European Coordinating Facility (ST-ECF/ESA), and the Canadian Astronomy Data Centre (CADC/NRC/CSA). ZEUS-2 development and observations are supported by NSF grants AST-0705256, AST-0722220, AST-1105874, and AST-1109476; NASA grant NNX10AM09H; and a grant from Georgia Southern University. C.F. thanks R. Wang for sharing her list of high- $z$ [C II] detections from the literature and in Wang et al. (2013). Last, the authors thank the anonymous referees for their helpful comments and acknowledge the APEX staff whose excellent support helped to make this work possible.

\section{REFERENCES}

Agertz, O., Teyssier, R., \& Moore, B. 2011, MNRAS, 410, 1391 Aravena, M., Bertoldi, F., Schinnerer, E., et al. 2008, A\&A, 491, 173 Barger, A. J., Cowie, L. L., \& Sanders, D. B. 1999, ApJL, 518, L5 Bertone, S., \& Conselice, C. J. 2009, MNRAS, 396, 2345 Bothwell, M. S., Smail, I., Chapman, S., et al. 2013, MNRAS, 429, 3047 Biggs, A. D., \& Ivison, R. J. 2008, MNRAS, 385, 893 Bradford, C. M., Nikola, T., Stacey, G. J., et al. 2003, ApJ, 586, 891 Brauher, J. R., Dale, D. A., \& Helou, G. 2008, ApJS, 178, 280 Brinchmann, J., Charlot, S., White, S. D. M., et al. 2004, MNRAS, 351, 1151 Brisbin, D., Ferkinnoff, C., Nikola, T., et al. 2013, ApJ, in press Carilli, C. L., Riechers, D., Walter, F., et al. 2013, ApJ, 763, 120 Colbert, J. W., Malkan, M. A., Clegg, P. E., et al. 1999, ApJ, 511, 721 Coppin, K., Chapin, E. L., Mortier, A. M. J., et al. 2006, MNRAS, 372, 1621 Coppin, K. E. K., Danielson, A. L. R., Geach, J. E., et al. 2012, MNRAS, 427,520

Cox, P., Krips, M., Neri, R., et al. 2011, ApJ, 740, 63

Crawford, M. K., Lugten, J. B., Fitelson, W., Genzel, R., \& Melnick, G. 1986, ApJL, 303, L57

Daddi, E., Dickinson, M., Morrison, G., et al. 2007, ApJ, 670, 156

De Breuck, C., Maiolino, R., Caselli, P., et al. 2011, A\&A, 530, L8 Decarli, R., Walter, F., Neri, R., et al. 2012, ApJ, 752, 2

Di Matteo, T., Khandai, N., DeGraf, C., et al. 2012, ApJL, 745, L29 Downes, D., \& Solomon, P. M. 1998, ApJ, 507, 615

Dye, S., Negrello, M., Hopwood, R., et al. 2013, MNRAS, submitted (arXiv:1311.5893)

Eales, S., Dunne, L., Clements, D., et al. 2010, PASP, 122, 499

Elbaz, D., Daddi, E., Le Borgne, D., et al. 2007, A\&A, 468, 33

Elbaz, D., Dickinson, M., Hwang, H. S., et al. 2011, A\&A, 533, A119

Ferkinhoff, C., Brisbin, D., Nikola, T., et al. 2011, ApJL, 740, L29

Ferkinhoff, C., Hailey-Dunsheath, S., Nikola, T., et al. 2010, ApJL, 714, L147

Fu, H., Cooray, A., Feruglio, C., et al. 2013, Natur, 498, 329

Gallerani, S., Neri, R., Maiolino, R., et al. 2012, A\&A, 543, A114 
Genel, S., Naab, T., Genzel, R., et al. 2012, ApJ, 745, 11

Genzel, R., Tacconi, L. J., Gracia-Carpio, J., et al. 2010, MNRAS, 407, 2091 Graciá-Carpio, J., Sturm, E., Hailey-Dunsheath, S., et al. 2011, ApJL, 728, L7

Hailey-Dunsheath, S., Nikola, T., Stacey, G. J., et al. 2010, ApJL, 714, L162

Helou, G., Khan, I. T., Malek, L., \& Boehmer, L. 1988, ApJS, 68, 151

Hildebrand, R. H., Loewenstein, R. F., Harper, D. A., et al. 1985, Icar, 64, 64

Hopkins, P. F., Croton, D., Bundy, K., et al. 2010, ApJ, 724, 915

Hughes, D. H., Serjeant, S., Dunlop, J., et al. 1998, Natur, 394, 241

Hung, C.-L., Sanders, D. B., Casey, C. M., et al. 2013, ApJ, 778, 129

Iono, D., Wilson, C. D., Yun, M. S., et al. 2009, ApJ, 695, 1537

Iono, D., Yun, M. S., Elvis, M., et al. 2006, ApJL, 645, L97

Israel, F. P. 2009, A\&A, 493, 525

Ivison, R. J., Swinbank, A. M., Swinyard, B., et al. 2010, A\&A, 518, L35

Joy, M., Lester, D. F., \& Harvey, P. M. 1987, ApJ, 319, 314

Kaufman, M. J., Wolfire, M. G., \& Hollenbach, D. J. 2006, ApJ, 644, 283

Kaufman, M. J., Wolfire, M. G., Hollenbach, D. J., \& Luhman, M. L. 1999, ApJ, 527,795

Keeton, C. R. 2001, arXiv:0102340

Keres, D., Katz, N., Weinberg, D. H., \& Dave, R. 2005, MNRAS, 363, 2

Kitzbichler, M. G., \& White, S. D. M. 2007, MNRAS, 376, 2

Leipski, C., Meisenheimer, K., Walter, F., et al. 2013, ApJ, 772, 103

Lord, S. D., Hollenbach, D. J., Haas, M. R., et al. 1996, ApJ, 465, 703

Lotz, J. M., Jonsson, P., Cox, T. J., et al. 2011, ApJ, 742, 103

Luhman, M. L., Satyapal, S., Fischer, J., et al. 1998, ApJL, 504, L11

Luhman, M., Satyapal, S., Fischer, J., et al. 2003, ApJ, 594, 758

Lupu, R. E., Scott, K. S., Aguirre, J. E., et al. 2012, ApJ, 757, 135

Maiolino, R., Caselli, P., Nagao, T., et al. 2009, A\&A, 500, L1

Maiolino, R., Cox, P., Caselli, P., et al. 2005, A\&A, 440, L51

Maiolino, R., Gallerani, S., Neri, R., et al. 2012, MNRAS, 425, L66

Malhotra, S., Kaufman, M. J., Hollenbach, D., et al. 2001, ApJ, 561, 766

Meijerink, R., Spaans, M., \& Israel, F. P. 2006, ApJL, 650, L103

Negishi, T., Onaka, T., Chan, K.-W., \& Roellig, T. L. 2001, A\&A, 375, 566

Negrello, M., Hopwood, R., De Zotti, G., et al. 2010, Sci, 330, 800
Negrello, M., Hopwood, R., Dye, S., et al. 2013, MNRAS, submitted (arXiv:1311.5898)

Noeske, K. G., Faber, S. M., Weiner, B. J., et al. 2007, ApJL, 660, L43

Oberst, T. E., Parshley, S. C., Stacey, G. J., et al. 2006, ApJL, 652, L125

Pety, J., Beelen, A., Cox, P., et al. 2004, A\&A, 428, L21

Pound, M. W., \& Wolfire, M. G. 2008, in ASP Conf. Ser. 394, Astronomical Data Analysis Software and Systems (San Francisco, CA: ASP), 654

Rice, W., Lonsdale, C. J., Soifer, B. T., et al. 1988, ApJS, 68, 91

Riechers, D. A., Bradford, C. M., Clements, D. L., et al. 2013, Natur, 496, 329

Sanders, D. B., \& Mirabel, I. F. 1996, ARA\&A, 34, 749

Sargsyan, L., Lebouteiller, V., Weedman, D., et al. 2012, ApJ, 755, 171

Siebenmorgen, R., \& Krügel, E. 2007, A\&A, 461, 445

Smail, I., Ivison, R. J., \& Blain, A. W. 1997, ApJL, 490, L5

Spergel, D. N., Verde, L., Peiris, H. V., et al. 2003, ApJS, 148, 175

Stacey, G. J., Geis, N., Genzel, R., et al. 1991, ApJ, 373, 423

Stacey, G. J., Hailey-Dunsheath, S., Ferkinhoff, C., et al. 2010, ApJ, 724,957

Sturm, E., Verma, A., Graciá-Carpio, J., et al. 2010, A\&A, 518, L36

Swinbank, A. M., Karim, A., Smail, I., et al. 2012, MNRAS, 427, 1066

Tacconi, L. J., Genzel, R., Neri, R., et al. 2010, Natur, 463, 781

Tacconi, L. J., Neri, R., Genzel, R., et al. 2013, ApJ, 768, 74

Tielens, A. G. G. M., \& Hollenbach, D. 1985, ApJ, 291, 722

Valtchanov, I., Virdee, J., Ivison, R. J., et al. 2011, MNRAS, 415, 3473

Venemans, B. P., McMahon, R. G., Walter, F., et al. 2012, ApJL, 751, L25

Wagg, J., Wiklind, T., Carilli, C. L., et al. 2012, ApJL, 752, L30

Wang, R., Wagg, J., Carilli, C. L., et al. 2013, ApJ, 773, 44

Ward, J. S., Zmuidzinas, J., Harris, A. I., \& Isaak, K. G. 2003, ApJ, 587, 171

Weiß, A., Downes, D., Walter, F., \& Henkel, C. 2005b, A\&A, 440, L45

Weiß, A., Kovács, A., Coppin, K., et al. 2009, ApJ, 707, 1201

Weiß, A., Walter, F., \& Scoville, N. Z. 2005a, A\&A, 438, 533

Willott, C. J., Omont, A., \& Bergeron, J. 2013, ApJ, 770, 13

Wolfire, M. G., Tielens, A. G. G. M., \& Hollenbach, D. 1990, ApJ, 358, 116 